\title{
Erratum to: ESUR recommendations for MR imaging of the sonographically indeterminate adnexal mass: an update
}

\author{
Rosemarie Forstner $^{1}$ - Isabelle Thomassin-Naggara ${ }^{2}$ - Teresa Margarida Cunha ${ }^{3}$. \\ Karen Kinkel $^{4}$ - Gabriele Masselli ${ }^{5}$. Rahel Kubik-Huch ${ }^{6}$. John A. Spencer ${ }^{7}$. \\ Andrea Rockall $^{8,9}$
}

Published online: 5 December 2016

(C) European Society of Radiology 2016

\section{Erratum to: Eur Radiol}

\section{DOI 10.1007/s00330-016-4600-3}

The name of the author Gabriele Masselli was rendered wrongly in the original publication, but has since been corrected. The publisher apologises for this error and the inconvenience caused.

The online version of the original article can be found at http://dx.doi. org/10.1007/s00330-016-4600-3.

Rosemarie Forstner

R.Forstner@salk.at

Isabelle Thomassin-Naggara

isabellethomassin@gmail.com

Teresa Margarida Cunha

tmargarida@gmail.com

Karen Kinkel

karen.kinkel@grangettes.ch

Gabriele Masselli

gabriele.masselli@uniroma1.it

Rahel Kubik-Huch

rahel.kubik@ksb.ch

Andrea Rockall

a.rockall@imperial.ac.uk

1 Department of Radiology, Landeskliniken Salzburg Paracelsus Medical University, Müllner Hauptstr. 48, A-5020 Salzburg, Austria
2 Sorbonne Universités, UPMC Univ. Paris 06, Institut Universitaire de Cancérologie, Assistance Publique - Hôpitaux de Paris (AP-HP), Hôpital Tenon, Service de Radiologie, 54 avenue Gambetta, 75020 Paris, France

3 Serviço de Radiologia, Instituto Portugues de Oncologia de Lisboa Francisco Gentil, R. Prof. Lima Basto, 1099-023 Lisboa, Portugal

4 Institut de Radiologie Clinique des Grangettes, Chemin des Grangettes 7, CH 1224 Chêne-Bougeries, Switzerland

5 Radiology Department, Sapienza University, Viale del Policlinico 155, 00161 Rome, Italy

6 Institut of Radiology, Departement of Medical Services, Kantonsspital Baden, Im Ergel, CH-5404 Baden, Switzerland

7 Department of Radiology, St James's University Hospital, Beckett Street, Leeds LS9 7TF, UK

8 Consultant Radiologist, The Royal Marsden Hospital NHS Foundation Trust, London, UK

9 Visiting Professor, Imperial College, London, UK 